\title{
High Frequency Boundary Layer Actuation by Fluidic Oscillators at High Speed Test Conditions
}

\author{
Valentin Bettrich, Martin Bitter and Reinhard Niehuis
}

\begin{abstract}
Detailed investigations of high frequency pulsed blowing and the interaction with the boundary layer at high speed test conditions were performed on a flat plate with pressure gradient. This experimental testbed features the imposed suction side flow of an aerodynamically highly loaded low pressure turbine profile. For actuation, a newly developed coupled fluidic oscillator with an independent mass flow and frequency characteristic was tested successfully. Several oscillator operating points were investigated at one turbine profile equivalent operating point with Reynolds number of 70,000, theoretical outflow Mach number of 0.6 , and an inflow free stream turbulence level of $4 \%$. The examined frequency range was between 6.5 and $7.5 \mathrm{kHz}$ and the actuation mass flow rates were varied between $0.68 \%$ and $1.32 \%$ of the overall passage mass flow. As a result, the flow separation and transition can be controlled and the suction side profile losses even halved. Differences in the interaction with the boundary layer of the different oscillator operating points are also presented and discussed.
\end{abstract}

Keywords Active flow control $\cdot$ Fluidic oscillator High frequency pulsed blowing • Boundary layer actuation Flat plate with pressure gradient $\cdot$ Aerodynamic loading Boundary layer transition $\cdot$ Flow separation

\footnotetext{
V. Bettrich $(\varangle) \cdot$ M. Bitter $\cdot$ R. Niehuis

Institute of Jet Propulsion, Bundeswehr University Munich, Neubiberg, 85577 Munich, Germany e-mail: valentin.bettrich@unibw.de URL:https://www.unibw.de/isa 


\section{Nomenclature}

\section{Symbols}

$\begin{aligned} h & {[\mathrm{~mm}] \text { wall-normal height } } \\ f & {[\mathrm{~Hz}] \text { frequency } } \\ \dot{m} & {[\mathrm{~kg} / \mathrm{s}] \text { mass flow rate } } \\ M a & {[-] \text { Mach number } } \\ p & {[\mathrm{~Pa}] \text { pressure (static in case of no subscript) } } \\ q & {[\mathrm{~Pa}] \text { dynamic pressure } } \\ R e & {[-] \text { Reynolds number } } \\ s & {[\mathrm{~mm}] \text { surface length } } \\ S r & {[-] \text { Strouhal number } } \\ t & {[\mathrm{~mm}] \text { spacing between two oscillator outlets } } \\ T u & {[\%] \text { turbulence level } } \\ U & {[\mathrm{~m} / \mathrm{s}] \text { velocity } } \\ V & {[\mathrm{~m} / \mathrm{s}] \text { velocity based on optical measurements } } \\ z & {[\mathrm{~mm}] \text { spanwise direction } } \\ \beta & {[-] \text { Hartree parameter } } \\ \delta * & \text { mm displacement thickness } \\ \delta_{2} & \text { mm momentum thickness } \\ \delta_{99} & \text { mm boundary layer thickness } \\ \gamma & {[-] \text { ratio of specific heats } } \\ \nu & {[\mathrm{m} / \mathrm{s}] \text { kinematic viscosity } } \\ \zeta & {[-] \text { total pressure loss coefficient } }\end{aligned}$

\section{Subscripts}

1 Inflow condition

2,th Theoretical exit condition

$\infty \quad$ Free stream condition

AFC Active flow control

inst. Instability

int Integral value

is Isentropic

osc Based on the oscillator

pas One passage of the cascade / experimental testbed

Pp Based on Preston probe

PIV Based on Particle Image Velocimetry

$\mathrm{t}$ Total condition

TEC Based on the turbine exit casing

tot Total surface length 
T161 Based on the T161 results

Wazzan Based on Wazzan's charts

Walker Based on Walker's equation

\section{Abbreviations}

$\begin{aligned} \text { AFC } & \text { Active Flow Control } \\ \text { CTA } & \text { Constant Temperature Anemometry } \\ \text { DEHS } & \text { Di-Ethyl-Hexyl-Sebacat } \\ \text { HGK } & \text { Hochgeschwindigkeits-Gitterwindkanal (High Speed Cascade Wind } \\ & \text { Tunnel) } \\ \text { ISA } & \text { Institut für Strahlantriebe (Institute of Jet Propulsion) } \\ \text { KH } & \text { Kelvin-Helmholtz } \\ \text { LPT } & \text { Low Pressure Turbine } \\ \text { PIV } & \text { Particle Image Velocimetry } \\ \text { PSD } & \text { Power Spectral Density } \\ \text { TS } & \text { Tollmien-Schlichting }\end{aligned}$

\section{Introduction}

Since the general research trend identified active flow control (AFC) to play a key role for efficient aerodynamic applications, many different concepts and methods were established. Depending on the flow problem or system to be controlled, different approaches are possible or reasonable. The important questions for a chosen flow problem arise regarding the most efficient actuator concept, the actuation position, the actuation impact or energy, and actuation frequency.

The latest flow control concepts are all "active". Among them, differences can be found in their actual mechanism principle. Some concepts use sensor feedback for controllers, e.g. closed-loop designs like in the work of King et al. [1]. Other applications are considered to be active if the actuator is switched on and off when needed, as outlined in the review paper by Niehuis and Mack [2]. In some cases, feedback control cannot be applied due to small geometries, high temperatures or frequencies, or if it is just the more expedient approach. The variety of available actuators is also quite high. The most important ones to name are plasma, piezo, and synthetic jet actuators as well as fluidic oscillators. Cattafesta [3] gives a comprehensive overview of the mentioned ones and considers even more actuators. For flow separation problems, many studies on the most efficient position of actuation were already carried out. A suggestion for an optimal actuation according to [4-8] is at the position of highest receptivity.

Considering mass flow investment, Mack et al. [9] used for AFC on the T161, an aerodynamically highly loaded low pressure turbine (LPT) research profile, fluidic 
oscillators with a mass flow rate related to the passage mass flow of $\dot{m}_{\text {osc., } T 161} \approx$ $1.14 \% \cdot \dot{m}_{\text {pas. }, T 161}$. To improve the efficiency, the latest investigations at the Institute of Jet Propulsion (ISA) prove for an aerodynamically highly loaded LPT exit casing profile that the mass flow investment of the oscillator can be decreased as low as $\dot{m}_{\text {osc. }, T E C}=2.1 \cdot 10^{-4} \cdot \dot{m}_{\text {pas. }, T E C}$. The key for this achievement is to consider the $\mathrm{AFC}$ in the design process with an optimized position and the right trigger frequency [10-12].

Which concept, actuator, position, impact, or frequency range should be used depends on many parameters, e.g. the velocity distribution, necessary actuation frequency or available reaction time, available sensors, controllers, and actuators as well as the nature of the flow problem-periodic or random. In turbomachinery, one main research area for flow control problems are LPTs. Modern LPTs are aerodynamically highly loaded, usually featuring flow separation on the suction side towards low Reynolds number operating points. Since the high speed investigations at ISA showed many promising results [2, 9, 13, 14] for AFC applied on the T161, this paper focuses on a T161-like suction side flow of a flat plate with pressure gradient. The massive flow separation is controlled with high frequency periodic excitation [15], induced by a newly developed coupled fluidic oscillator [16].

The focus of this paper are investigations of different oscillator operating points and their respective influence on loss behavior, transition and interaction with the boundary layer. According to literature it is most promising to actuate in the range of the natural instabilities or receptivity of the boundary layer. Regarding the discussion whether to trigger the so-called "Tollmien-Schlichting" (TS) or the "KelvinHelmholtz" (KH) instabilities, a simple explanation might clarify the topic. The TS waves or instabilities develop naturally in the laminar boundary layer and can therefore triggered [17]. The instabilities developing and growing within the shear layer for separated flows are commonly known as KH instabilities [18]. Consequently, the goal is to trigger with the most effective frequency in the range of the boundary layer receptivity to amplify linear instability effects and therefore use this interaction within the boundary layer for a controlled transition towards a turbulent boundary layer.

\section{Experimental Setup}

\subsection{Actuator-Coupled Fluidic Oscillator}

The actuator employed for the investigations of this paper is a coupled fluidic oscillator developed at ISA [16]. In the experimental testbed (Sect. 2.3) an array of actuators enables distributed pulsed blowing at very high frequencies of several $\mathrm{kHz}$. The unique advantage of the coupled oscillator over all common designs is the ability to change frequency and mass flow independently in a certain range, which is fundamental for detailed investigations of the impact and interaction between the boundary layer and the AFC device. 

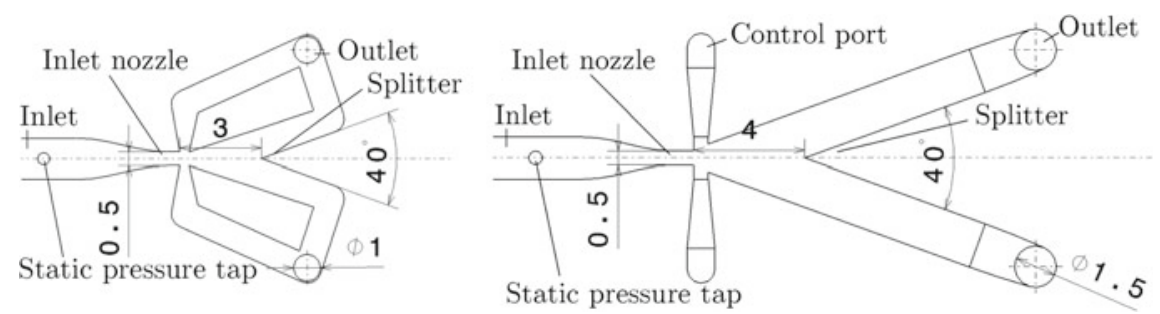

Fig. 1 Master oscillator (left) and slave oscillator (right) (all dimensions in millimeter)

The frequency of the coupled fluidic oscillator can be tuned with a standard feedback oscillator (Fig. 1 left). This design, which has a direct dependency between mass flow rate and frequency, was already used in former studies (see $[2,9,13,14]$. In contrast to the former applications, the pulsed blowing at the outlet of the feedback oscillator (master) is not directly used for flow control, but for frequency tuning of the oscillator without feedback loops (slave) through the control ports (Fig. 1 right). The excitation of the master forces the additional or secondary mass flow entering the system through the slave's inlet nozzle to flip according to the trigger frequency to each side of the splitter. The combined mass flow leaves the device as high frequency pulses through the outlets of the slave oscillator at the master's frequency. More details on the working principle of the coupled fluidic oscillator and a characterization of the actuator pulses at different operating points can be found in [16].

\subsection{High Speed Cascade Wind Tunnel}

The results presented here are based on experiments performed in the High Speed Cascade Wind Tunnel (HGK) at ISA of the Bundeswehr University Munich. The core of the test facility (Fig. 2) is an open loop wind tunnel which is placed inside a $12 \times 4 \mathrm{~m}$ pressure tank. A six stage axial compressor is driven

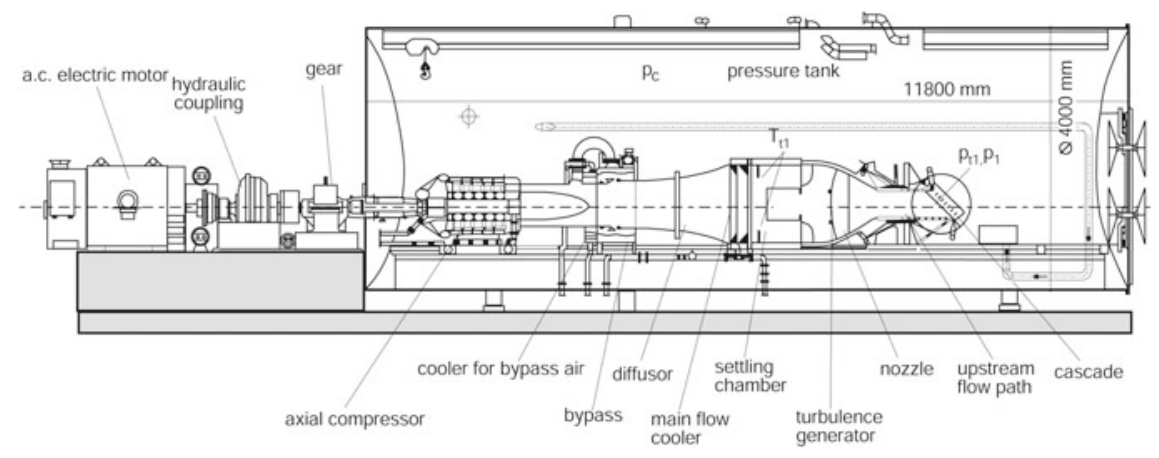

Fig. 2 High speed cascade wind tunnel (HGK) test facility 
by an external 1.3 MW a.c. electric motor. It enables compression ratios up to 2.14. The operating point is adjusted with a variation in shaft speed, a variable bypass, and coolers. Engine relevant turbulence levels can be realized with a turbulence generator just upstream the nozzle. In the pressure tank it is possible to vary the absolute pressure level from 35 mbar to approximately 1200 mbar. The independent variation of the Reynolds and Mach number makes the HGK test facility very unique among very few world wide. More details can be found in Sturm and Fottner [19].

For the specific experimental testbed boundary layer suction at the contoured walls (Sect. 2.3) of the configuration was required. This was realized with the secondary air supply system of the test facility. A one-stage radial compressor sucks at the test section inside the pressure tank and re-injects it into the vessel, in order to preserve the over all pressure level.

\subsection{Flat Plate with Pressure Gradient}

Motivated by several promising investigations regarding passive and AFC concepts on the aerodynamically highly loaded T161 LPT cascade, summarized by Niehuis and Mack [2], the research on this topic with the well-known boundary layer topology was intensified. The T161 low pressure turbine profile features a rather large separation bubble at low and even an open separation at very low Reynolds numbers. Therefore, it is an ideal experimental testbed to investigate flow control concepts in this Reynolds number regime. However, with the use of the coupled fluidic oscillator for the planned fundamental investigations, the T161 blade geometry is physically too small to integrate the new actuator system inside the cascade profile at its recent design stage. That is why a flat plate with pressure gradient, imposed by symmetric contoured walls, was developed in order to generate a closely matching boundary layer topology at the same flow conditions as for the T161 profile. The benefit of the new configuration is also a better accessibility for probes and optical measurement techniques. The experimental testbed integration into the HGK test facility is shown in Fig. 3. The flat plate itself (Fig. 4) consists of three individual plates. The uppermost one (yellow) features a super elliptical leading edge, static pressure taps, and the oscillator outlets. The slave oscillator array is integrated underneath in the middle plate (orange) and is fed with air through the plenum. The actuators for the frequency adjustment (master oscillators) are deployed in the lowermost plate (red), also separately supplied with air through the master's plenum. Through the slave oscillator outlets, the combined mass flow enters the boundary layer as high frequency pulsed blowing. Static pressure taps just upstream the experimental testbed in the wind tunnel side walls are used to ensure homogeneous inflow conditions. Furthermore, the contoured walls were also equipped with static pressure taps in both passages to ensure symmetric main flow conditions. The homogeneous and symmetric inflow conditions are verifiable not affected with activated oscillator on the upper surface of the flat plate only. Thus, equivalent passage inflow conditions can be assumed for all cases. In order to control the main passage flow and to prevent separation on the 
Fig. 3 Experimental testbed for the cascade section of the HGK

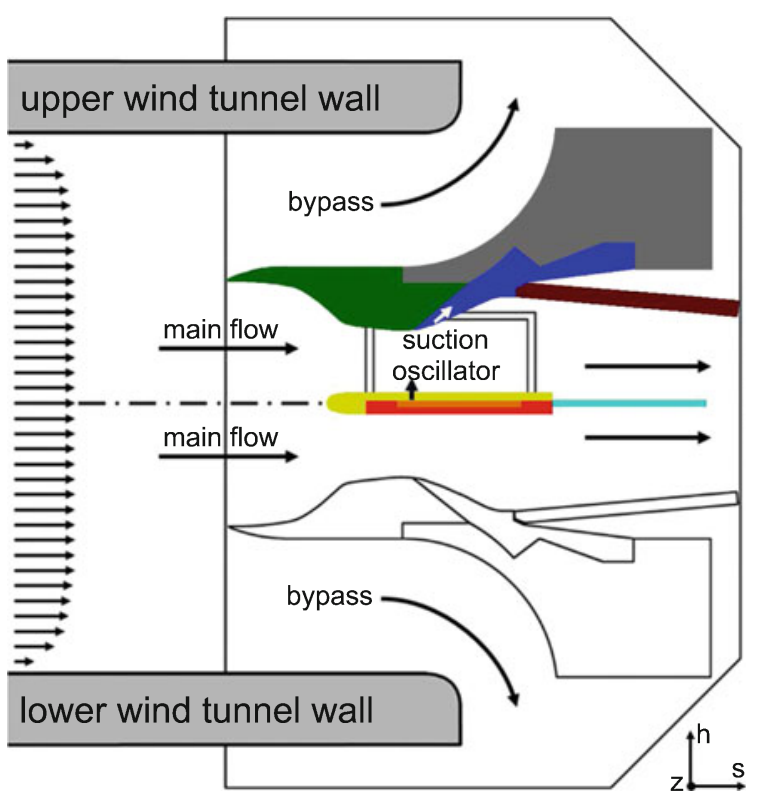

Fig. 4 Detailed view of the experimental testbed with the coupled fluidic oscillators

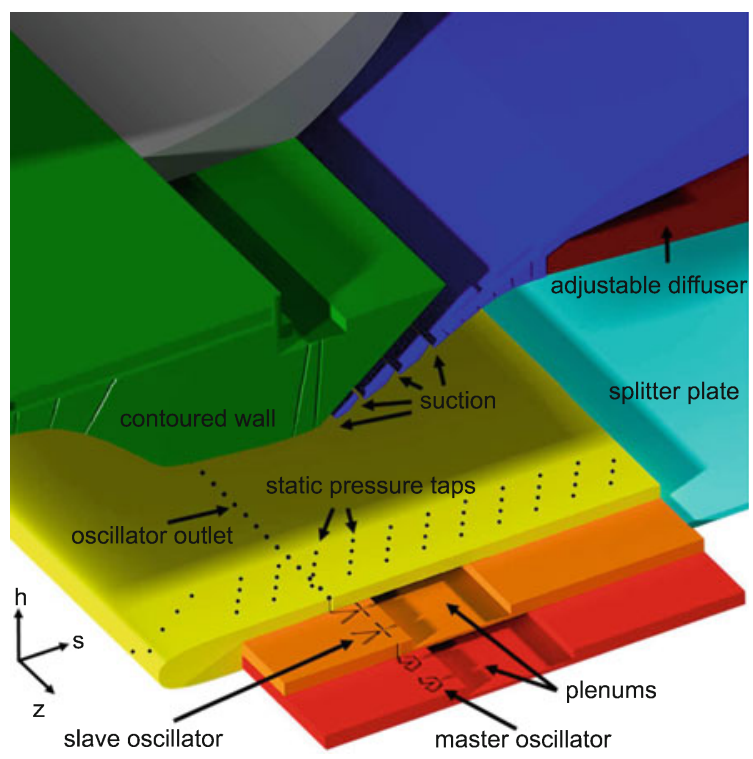


outer contour, air is sucked by several slots installed on contoured walls, opposing the flat plate. A splitter was assembled downstream to avoid vortex shedding at the trailing edge of the flat plate. The adjustable diffuser is used to fine-tune the flat plate boundary layer characteristic. More details and aspects of the design process can be found in [15].

\section{Instrumentation and Data Acquisition}

\subsection{Surface Pressure Distribution}

Compared to the T161 profile, the size of the flat plate is a 1:1 suction side surface length scale. It is equipped with 33 static pressure taps within $s / s_{\text {tot }}=0$ and 1 , ensuring twice the resolution of static pressure taps in relation to the T161 cascade, compare $[2,9,13,14]$. The positions $s / s_{\text {tot }}=0$ and 1 correspond to the leading and trailing edge, respectively. The pressure distribution is presented as the isentropic Mach number distribution in relation to the flat plate's surface length $s$

$$
M a_{i s}\left(s / s_{t o t}\right)=\sqrt{\frac{2}{\gamma-1} \cdot\left[\left(\frac{p_{t 1}}{p\left(s / s_{t o t}\right)}\right)^{\frac{\gamma-1}{\gamma}}-1\right]} .
$$

At the inlet of the test section, the total pressure $p_{t 1}$ is measured with a pitot tube. The isentropic Mach number along the surface is calculated with local static pressure data $p\left(s / s_{\text {tot }}\right)$, attained from the static pressure taps. As part of the standard measurement equipment, a 98RK rack mounted pressure system is used to acquire the pressure data. For the presented results pressure transducers with 345 mbar full scale range (uncertainty of $0.05 \%$ ) were used. Hence, the resulting uncertainty of the Mach number is less than $\Delta M a_{i s} \leq 0.02$ for the investigated operating point.

\subsection{Preston Probe Measurements}

A Preston probe, which is a flattened Pitot tube, was used to traverse closely along the surface. It is used here also for total pressure boundary layer traverses normal to the surface. The local dynamic pressure $q\left(s / s_{t o t}, h\right)$ is calculated with the local static pressure data $p\left(s / s_{t o t}\right)$ and the total pressure of the probe $p_{t}\left(s / s_{t o t}, h\right)$. It is normalized by the local dynamic pressure outside of the boundary layer $q_{\infty}\left(s / s_{t o t}\right)$ to obtain the dimensionless local dynamic pressure coefficient

$$
\frac{q\left(s / s_{t o t}, h\right)}{q_{\infty}\left(s / s_{t o t}\right)}=\frac{p_{t}\left(s / s_{t o t}, h\right)-p\left(s / s_{t o t}\right)}{p_{t 1}-p\left(s / s_{t o t}\right)} .
$$


More details on the Preston probe head shape and size, the procedure to determine the boundary layer condition (laminar, turbulent or separated), and the determination of the transition point are given in $[15,22]$.

Furthermore, the total pressure loss coefficient calculated from the Preston probe data is used to compare different actuator operating points. Therefore, boundary layer traverses were taken at $s / s_{\text {tot }}=1$, representing the trailing edge of the T161 profile. The total pressure loss coefficient is calculated by

$$
\zeta=\frac{p_{t 1}-p_{t}\left(s / s_{t o t}, h\right)}{p_{t 1}-p\left(s / s_{t o t}\right)} .
$$

In order to cover the entire boundary layer up to the free stream for all operating points, the integral value is calculated for $h=0$ to $15 \mathrm{~mm}$ by

$$
\zeta_{i n t}=\int_{h=0}^{h=15} \zeta(h) .
$$

A PSI 9116 pressure transducer with a full scale range of 69 mbar and $0.05 \%$ full scale range uncertainty was used for all Preston probe measurements. This results in an uncertainty of the total pressure loss coefficient of $\Delta \zeta \leq 0.005$ and a Mach number uncertainty of $\Delta M a \leq 0.01$ for the traverses at the edge of the boundary layer. It has to be noted that the uncertainty increases towards lower Mach numbers near the surface.

\subsection{Particle Image Velocimetry}

A standard planar two-component particle image velocimetry setup (2D2C-PIV) was realized for the characterization of the boundary layer topology with and without activated flow control mechanism. Two $4 \mathrm{Mpx}$ scientific CCD cameras were used side-by-side to measure the flow field across the full length of the flat plate in parallel. The mid plane of the configuration was illuminated by a Beamtech Vlite 200 double pulse Nd:YAG laser with $200 \mathrm{~mJ}$ pulse energy. A carefully polished model surface enabled the investigation of the wall-near velocity field down to sub-millimeter scale as diffuse reflections of the laser light on the model surface were minimized. This approach enables detailed near-wall PIV measurements [23]. The PIV tracer particles were produced from Di-Ethyl-Hexyl-Sebacat (DEHS) oil, generated in a Laskinnozzle seeding atomizer which typically produces particles with $1 \mu$ in size.

For the data processing, each PIV image was pre-processed with a shift correction to compensate for low frequency vibrations and by subtracting the local image minimum for intensity normalization. A Butterworth temporal filtering combined with a horizontal FFT-filtering allowed a complete suppression of the laser light reflection on the polished metal surface. Unfortunately, some tracer particles always remain stationary on the model surface even after careful cleaning. These particles partly 
impose spurious vectors to the final result at these positions at the wall. Nevertheless, a common multi-pass PIV with interrogation windows down to 16-by-16 pixel with $50 \%$ overlap was possible. As the particles had an imaged diameter of roughly 2 pixels, the final result was not effected by typical PIV bias errors as peak-locking. Finally, 2000 instantaneous vector fields were averaged. This evaluation procedure led to an effective spatial resolution of about $0.3 \mathrm{~mm}$ per velocity vector.

\subsection{Hot-Wire Boundary Layer Measurements}

For time-resolved data, hot-wire measurements were performed with a $1 \mathrm{D}$ hot-wire boundary layer probe (Type 55P15) from DANTEC, operated in the constant temperature anemometry (CTA) mode. A velocity calibration was conducted for the expected static pressure and velocity range. At each position the signal was acquired for $10 \mathrm{~s}$ at $60 \mathrm{kHz}$ with a $30 \mathrm{kHz}$ hardware low pass filter set to prevent aliasing effects. Based on the velocity data, the power spectral density (PSD) according to Welch's method, as implemented in Matlab with default inputs, is calculated.

The uncertainty of the hot-wire measurements is strongly dependent on the Reynolds number (static pressure within the vessel) and the flow velocity. At the absolute pressure level of the conducted measurements, the uncertainty for the velocity data is around $\pm 2 \%$ at the edge of the boundary layer. Due to a low thermal conduction as a consequence of low absolute static pressure of the operating point and the low velocities near the surface, the uncertainty increases within the boundary layer.

\section{Design Aspects of the Experimental Testbed}

\subsection{Validation of the Experimental Testbed and Oscillator Integration}

To ensure comparability with former results, the experimental testbed was validated against the T161 cascade measurements. Bettrich et al. [15] proved very good agreement between the flat plate with pressure gradient and the T161 suction side flow. The operating point was chosen at Reynolds number $R e=70,000$, theoretical outflow Mach number of $M a_{2, t h}=0.6$, and an inflow free stream turbulence level of $T u \approx 4 \%$. The suction side flow is imposed on a flat plate in 1:1 scale of the surface length. With matching in- and outlet conditions and without actuation, an excellent correspondence with former studies is shown, compare [9]. The Mach number distribution within the area of interest (white shaded) is shown in Fig. 5. The differences near the leading edge are typical for any flat plate setup, as discussed in [15]. Altogether, the design goals of the experimental testbed for the planned investigations is fully achieved. 


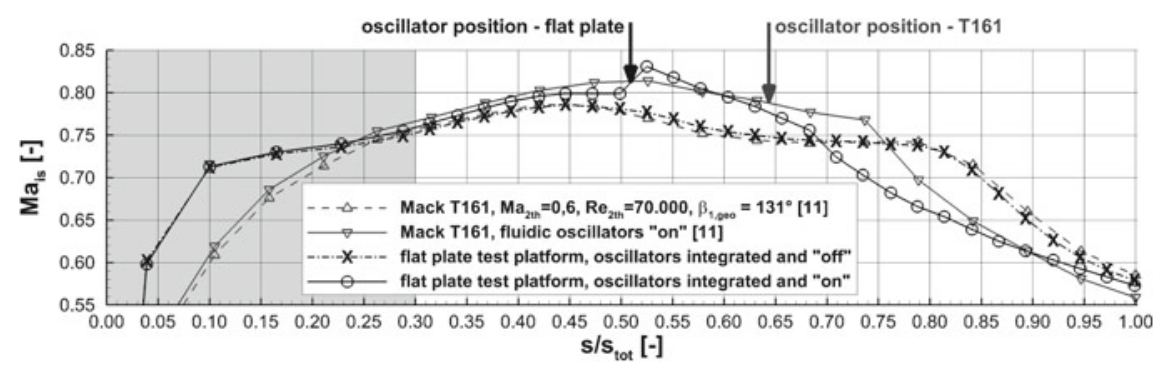

Fig. 5 Mach number distributions from static pressure taps

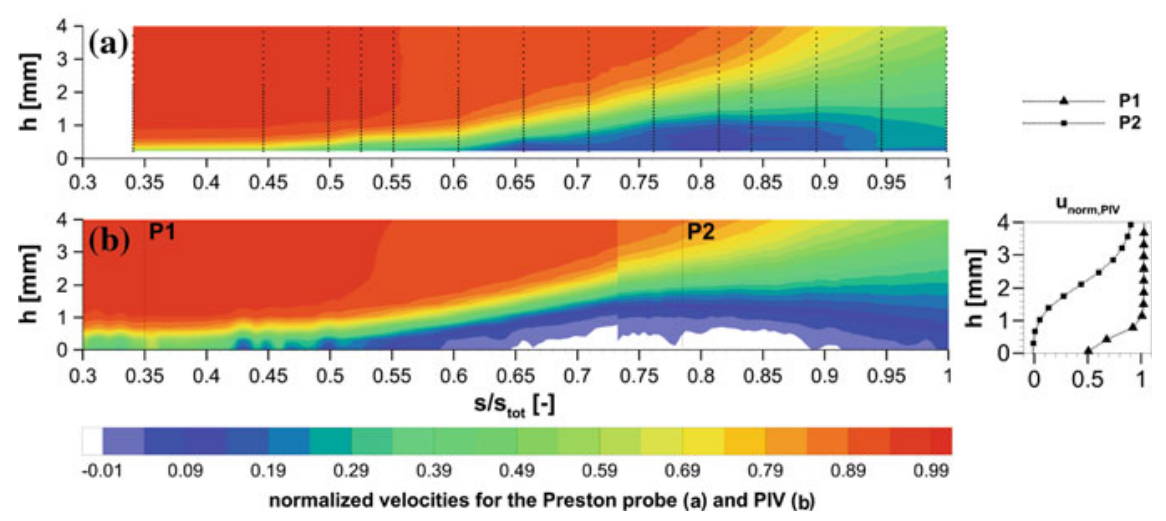

Fig. 6 Non-actuated boundary layer flow with separation bubble of the experimental testbed, based on Preston probe $\mathbf{a}$ and PIV $\mathbf{b}$ measurements

In order to resolve the boundary layer flow of the experimental testbed without actuation, Preston probe (Fig. 6a — each dot indicating a measured position) and PIV (Fig. 6b) measurements were performed. Both methods indicate the non-actuated time averaged flow field near the surface. While the overall flow field shows very good agreement, some differences in the separation bubble and the wall near flow are evident. Reason for differences are that only PIV can resolve reverse flow within the separation bubble (grey and white contours) but are quite challenging in high speed applications near the surface. The pneumatic investigations, acquired with the Preston probe, deliver very robust results, but allow only for limited resolution due to the size of the probe head, as discussed in [15].

In contrast to former studies with the T161 cascade, the oscillator outlets were positioned further upstream on the flat plate configuration. This approach was motivated by other results found in literature. It is proven to be beneficial in terms of mass flow investment (reduction of 60\%) and control effectiveness compared to the further downstream position, used in the T161 investigations [15]. 


\subsection{Estimation of Receptivity of the Boundary Layer}

The design goal for the coupled fluidic oscillator is to cover the range of receptivity. Since a precise determination of the actual instability bandwidth is a rather challenging problem, especially for high speed applications with very thin boundary layers, several empirical approaches were available and conducted. At first, the latest numerical data for the T161 cascade reveal an optimal actuation frequency around 7.6-7.8 kHz. Second, an estimation for the TS instabilities was carried out with the use of the spatial and temporal stability charts according to Wazzan et al. [20]. The charts indicate the temporal amplification rates for TS waves for different flow profiles from stagnation to separation and are attained by a step-by-step integration method of the Orr-Sommerfeld equations. Considering the case for separated flow (Hartree parameter $\beta=-0.1988$ ) and based on the boundary layer data, which differ slightly depending whether to use the Preston probe or PIV boundary layer data, it delivers a frequency range of $7 \mathrm{kHz} \leq f_{T S \text {, Wazzan }} \leq 8.5 \mathrm{kHz}$ for the separated flow. The third approach utilizes the correlation of Walker and Gostelow [21]:

$$
f_{T S, \text { Walker }}=\frac{3.2 \cdot U_{\infty}^{2}}{2 \pi \nu\left(\frac{U_{\infty} \delta^{*}}{\nu}\right)^{1.5}}=\frac{3.2 \cdot U_{\infty}^{0.5}}{2 \pi \nu^{-0.5} \cdot\left(\delta^{*}\right)^{1.5}} .
$$

The free stream velocity $U_{\infty}$ outside the boundary layer and the kinematic viscosity $\nu$ are straight-forward to determine, whereas the displacement thickness $\delta^{*}$ is very sensitive to the results of the boundary layer measurements. Calculating $\delta^{*}$ with the Preston probe measurements results in $f_{T S, \text { Walker, } P p} \approx 7.5 \mathrm{kHz}$ and with the PIV data in $f_{T S, \text { Walker, } P I V} \approx 6.8 \mathrm{kHz}$. A slight variation of the boundary layer profile results also in a change in displacement thickness, which has a strong influence on the potential instability frequency (compare Eq.5). Therefore the results of the different measurement techniques should be considered supplemental rather than only one of the two. Furthermore, the frequency estimation is also quite sensitive to the actual position of the separation point, since its location is used as reference for the variables and their values included in the equation. For details to determine the separation point see [15]. To conclude, the target actuation frequency for the oscillator is determined to be between 6.8 and $8.5 \mathrm{kHz}$.

\subsection{Design of the Fluidic Oscillator}

For the design of the coupled fluidic oscillator array, several requirements had to be taken into account. Most important, the frequency range of the oscillator should cover the receptive range of the boundary layer. The entire estimated frequency range at the low absolute pressure conditions with an adequate mass flow variability could not be realized with one actuator. Therefore, the focus was set on the lower half of the estimated range, because it appeared to be the most promising compromise. 
The mass flow rate of the coupled actuator was aimed to be comparable to the ones used for the T161 cascade measurements. This allows a comparison of the further upstream outlet position of the oscillator with former results. Furthermore, lower and higher mass flow rates should also be achieved.

The influence of the design parameters on the frequency range, the operating stability, and the mass flow rate was determined in preliminary experimental investigations. The design of the coupled actuator was performed in a two step process. First, the master oscillator was scaled to reach the desired frequency range. Second, the mass flow range was adjusted with the slave oscillator and the same outlet diameter of the actuator was chosen as for the T161 cascade measurements.

\section{Results and Discussion}

\subsection{Investigated Actuator Operating Points}

Within the coupled oscillator's operating range, several operating points were investigated. The frequency range was varied between 6.5 and $7.5 \mathrm{kHz}$ at a constant mass flow rate of $\dot{m}_{\text {osc. }} \approx 1.05 \% \cdot \dot{m}_{\text {pas. }}$. In addition, the mass flow rate was varied between $\dot{m}_{\text {osc }} \approx 0.78 \%$ and $1.32 \% \cdot \dot{m}_{\text {pas }}$. for a constant frequency of $7 \mathrm{kHz}$. An evaluation of the performance of each operating point can be derived from the total pressure loss coefficient (Fig. 7) and transition (Fig. 8, same legend as Fig. 7) behavior. Since the differences in the distribution of the total pressure loss coefficient between all operating points with activated actuation is rather small, the integral values $\zeta_{\text {int }}$ in wall-normal direction for $h=0$ to $15 \mathrm{~mm}$ are outlined in Fig. 7, too. The distribution

Fig. 7 Distribution and integral value of the total pressure loss coefficient for different operating points at $s / s_{\text {tot }}=1$ from Preston probe

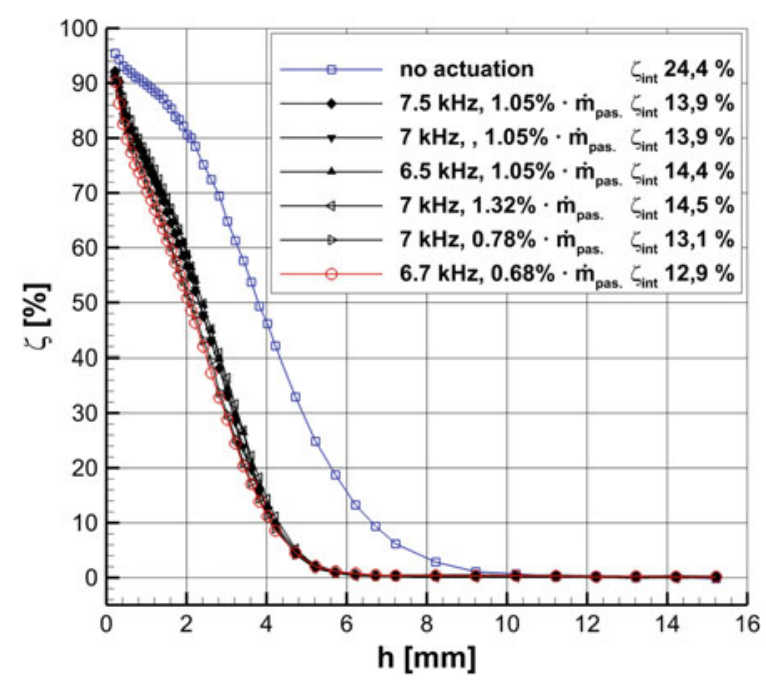


Fig. 8 Actuation effect on the boundary layer state for different actuator operating points (same legend as Fig. 7) from Preston probe

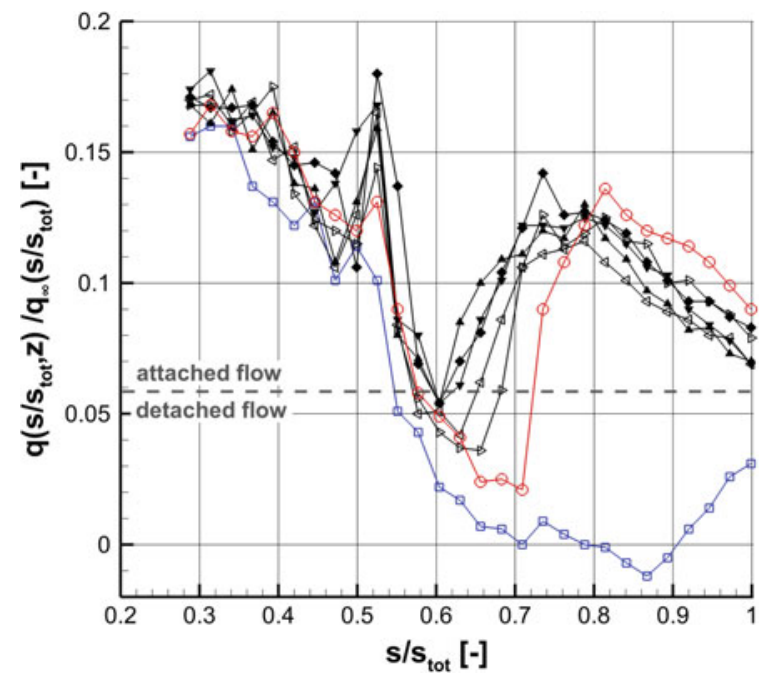

of the dimensionless local dynamic pressure coefficient along the surface (Fig. 8) indicates if separation occurs (values in the range of detached flow). It reveals as well if and where the transition takes place. Decreasing values can either represent a laminar or turbulent boundary layer, whereas a strong increase is an indicator for transition, compare Stotz et al. [22]. According to Fig. 8, for all actuated cases a transition to turbulent flow occurs. For some operating points even a small separation bubble is present. The impact of the oscillator can clearly be seen in all cases with actuation at $s / s_{\text {ges }} \approx 0.52$ (Fig. 8). If no actuation is applied, the boundary layer remains detached until the trailing edge.

It can clearly be seen in Figs. 7 and 8 that the frequency variation within the investigated range at constant mass flow rate is of minor importance on both loss behavior and transition. The key factor, however, is the mass flow investment. A reduction results in lower integral losses, as long as the open flow separation can be suppressed. Hence, the momentum itself is not the driving factor for loss reduction. For the constant frequency of $7 \mathrm{kHz}$ the transition point first moves upstream with increased mass flow rate and the integral total pressure losses increase accordingly. A further increase in mass flow rate then shifts the transition point downstream with a further increased loss characteristic. Thus, another mechanism aside the injected momentum has an influence on transition and loss behavior, which will be highlighted in Sect. 5.2.

Based on the observations drawn from the loss and transition behavior, the actuator operating point at the lowest overall mass flow rate $\left(\dot{m}_{\text {osc }} \approx 0.68 \% \cdot \dot{m}_{\text {pas. }}\right)$ and $6.7 \mathrm{kHz}$ actuation frequency was found to be most efficient in terms of mass flow investment and integral losses. Therefore, it was of special interest for a more fundamental investigation. It features a small and closed separation bubble, the furthest downstream transition point, and therefore the shortest turbulent boundary layer in 

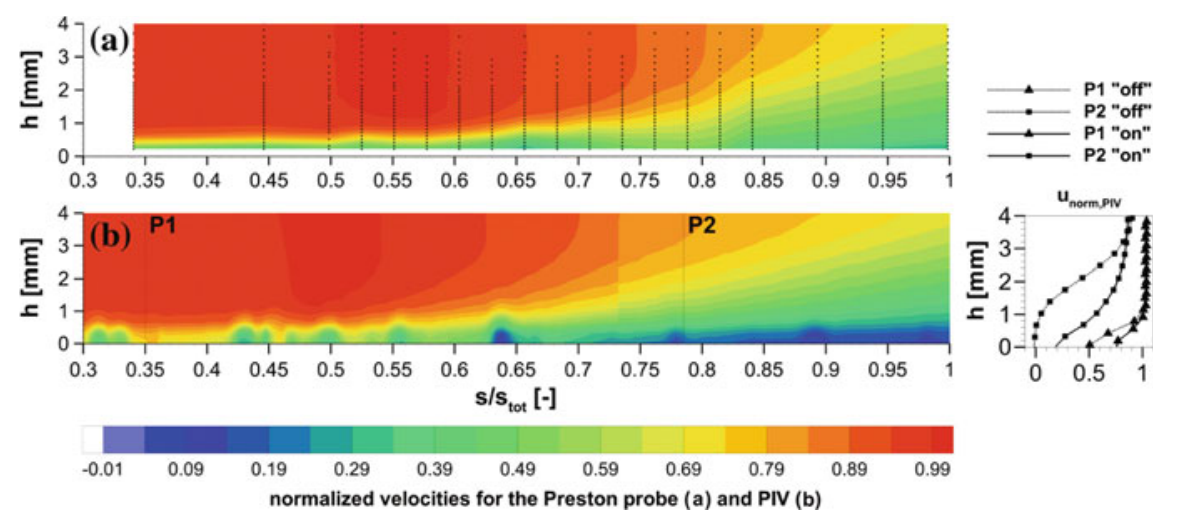

Fig. 9 Actuated boundary layer flow from Preston probe and PIV measurements for the $6.7 \mathrm{kHz}$ and $\dot{m}_{\text {osc }} \approx 0.68 \% \cdot \dot{m}_{\text {pas. }}$ operating point

streamwise extent. In contrast to the case without any AFC (Fig. 6), the controlled boundary layer topology is very different (Fig.9). The large separation bubble is successfully suppressed by the actuation. Both Figs. 6 and 9 were normalized with the same scales. Therefore, the peak Mach number increases with actuation applied, due to the reduced blocking effect. This can also clearly be seen in the surface Mach number distribution for both the flat plate with pressure gradient and the T161 cascade measurements, depicted in Fig. 5. The Preston probe and the PIV measurements were performed in streamwise direction in the centerline between two oscillator outlets. The steady and unsteady interaction phenomena of the $6.7 \mathrm{kHz}$ actuation with the boundary layer flow is already discussed in a previous paper [15]. A comparison of the interaction of different oscillator operating points with the boundary layer will be outlined below.

\subsection{High Frequency Pulsed Blowing Boundary Layer Interaction}

For the different oscillator operating points, 1D hot-wire measurements were carried out to investigate the respective interaction. Interaction is defined to an influence of the actuation pulse on the boundary layer flow. It takes place when the coherent structures, induced by the high frequency actuation, are not immediately damped away but can develop downstream and in wall-normal direction. If interaction takes place, the design goal of the AFC concept is reached and an increased power level in the spectrum must become visible. The results are presented in Fig. 10. It shows normalized PSD intensity plots in streamwise direction, parallel to the surface, for different wall-normal distances. The path for the hot-wire probe in streamwise direction was chosen to be in line with an oscillator outlet. The most upstream measurement posi- 
tion $s / s_{\text {ges }} \approx 0.5$ is just upstream of the oscillator outlet. The investigations cover positions at each static pressure tap all the way downstream to $s / s_{\text {ges }}=1$ and in wall normal distances of $h=0.2,0.6,1.0$, and $1.4 \mathrm{~mm}$. The frequency range shown is $500 \mathrm{~Hz}$ each, shifted for every operating point to visualize the oscillator influence and boundary layer interaction at a high level of detail. Each case is normalized with its highest PSD value for better comparison of the relative interaction phenomena. The individual highest PSD values differ only slightly amongst each other.

The normalized PSD plots in Fig. 10 reveal a strong interaction at all presented wall-normal distances for the $6.7 \mathrm{kHz}$ and $\dot{m}_{\text {osc }} \approx 0.68 \% \cdot \dot{m}_{\text {pas }}$ as well as the $7.0 \mathrm{kHz}$ and $\dot{m}_{o s c} \approx 0.78 \% \cdot \dot{m}_{\text {pas }}$ operating points. A decreased interaction of the actuation frequency with the boundary layer flow can be noticed for the $6.5 \mathrm{kHz}$ and $\dot{m}_{\text {osc }} \approx$ $1.05 \% \cdot \dot{m}_{\text {pas }}$ as well as for $7.5 \mathrm{kHz}$ and $\dot{m}_{\text {osc }} \approx 1.05 \% \cdot \dot{m}_{\text {pas }}$. A weak or damped interaction downstream the oscillator outlet and in wall-normal direction is present for the $6.5 \mathrm{kHz}$ and $\dot{m}_{\text {osc }} \approx 1.05 \% \cdot \dot{m}_{\text {pas }}$ as well as the $7.0 \mathrm{kHz}$ and $\dot{m}_{\text {osc }} \approx 1.32 \%$. $\dot{m}_{\text {pas }}$. The reason for the different degrees of interaction can either be a matter of the frequency, the initial momentum, or a feature of the oscillator itself. Since the pulsed flow of the oscillator can be considered constant flow superimposed by a (temporal) sinusoidal flow portion, higher mass flow rates at constant frequency can either result in a higher constant flow with relatively lower sinusoidal flow amplitudes or vise versa. However, these observations correspond well with the integral of the total pressure loss coefficients, presented in Fig. 7. Besides the fact of the different transition points, the lowest integral total pressure losses correspond to the strongest interaction with the boundary layer $\left(6.7 \mathrm{kHz}\right.$ and $\dot{m}_{o s c} \approx 0.68 \% \cdot \dot{m}_{\text {pas }}$ case).

With the interaction being identified as the second key factor of the AFC besides the induced momentum, the discrepancy of the transition point of the three different mass flow rates at $7 \mathrm{kHz}$ of Sect. 5.1 can be explained. For the $\dot{m}_{o s c} \approx 0.78 \% \cdot \dot{m}_{\text {pas }}$ case the mass flow rate is low but the interaction strong. Thus a further downstream but controlled transition can be achieved. Increasing the mass flow rate to $\dot{m}_{\text {osc }} \approx 1.05 \% \cdot \dot{m}_{\text {pas }}$, some interaction within the boundary layer can be observed. The higher momentum in combination with the boundary layer interaction consequently moves the transition point upstream. Increasing the momentum even further to $\dot{m}_{\text {osc }} \approx 1.32 \% \cdot \dot{m}_{\text {pas }}$, barely any interaction is noticed. Considering the delayed transition point compared to the $\dot{m}_{o s c} \approx 1.05 \% \cdot \dot{m}_{\text {pas }}$ case leads to the conclusion that the interaction has also a strong influence on transition control. A variation of the frequency $(6.5,7$, and $7.5 \mathrm{kHz})$ at the same mass flow rate of $\dot{m}_{\text {osc }} \approx 1.05 \% \cdot \dot{m}_{\text {pas }}$ shows that the interaction with the boundary layer for constant mass flow rates increases with increasing frequency. The differences in the integral total pressure losses confirm, that the profile losses depend not only on momentum but also on the degree of interaction of the boundary layer with the actuation. Among theses three oscillator operating points, the $6.5 \mathrm{kHz}$ case with the weakest interaction has higher integral losses than the other two cases.

To investigate the interaction in more detail, boundary layer traverse measurements were carried out for the most efficient oscillator operating point $(6.7 \mathrm{kHz}$ and $\left.\dot{m}_{\text {osc }} \approx 0.68 \% \cdot \dot{m}_{\text {pas }}\right)$ in terms of mass flow investment, loss characteristic, and interaction with the boundary layer. The results are presented in Fig. 11. It shows PSD 


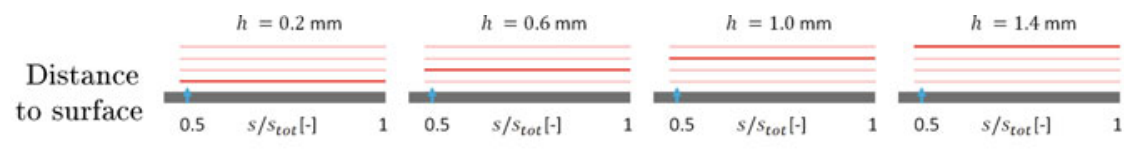

Operating

point

$6.7 \mathrm{kHz}$
$0.68 \% \dot{m}$
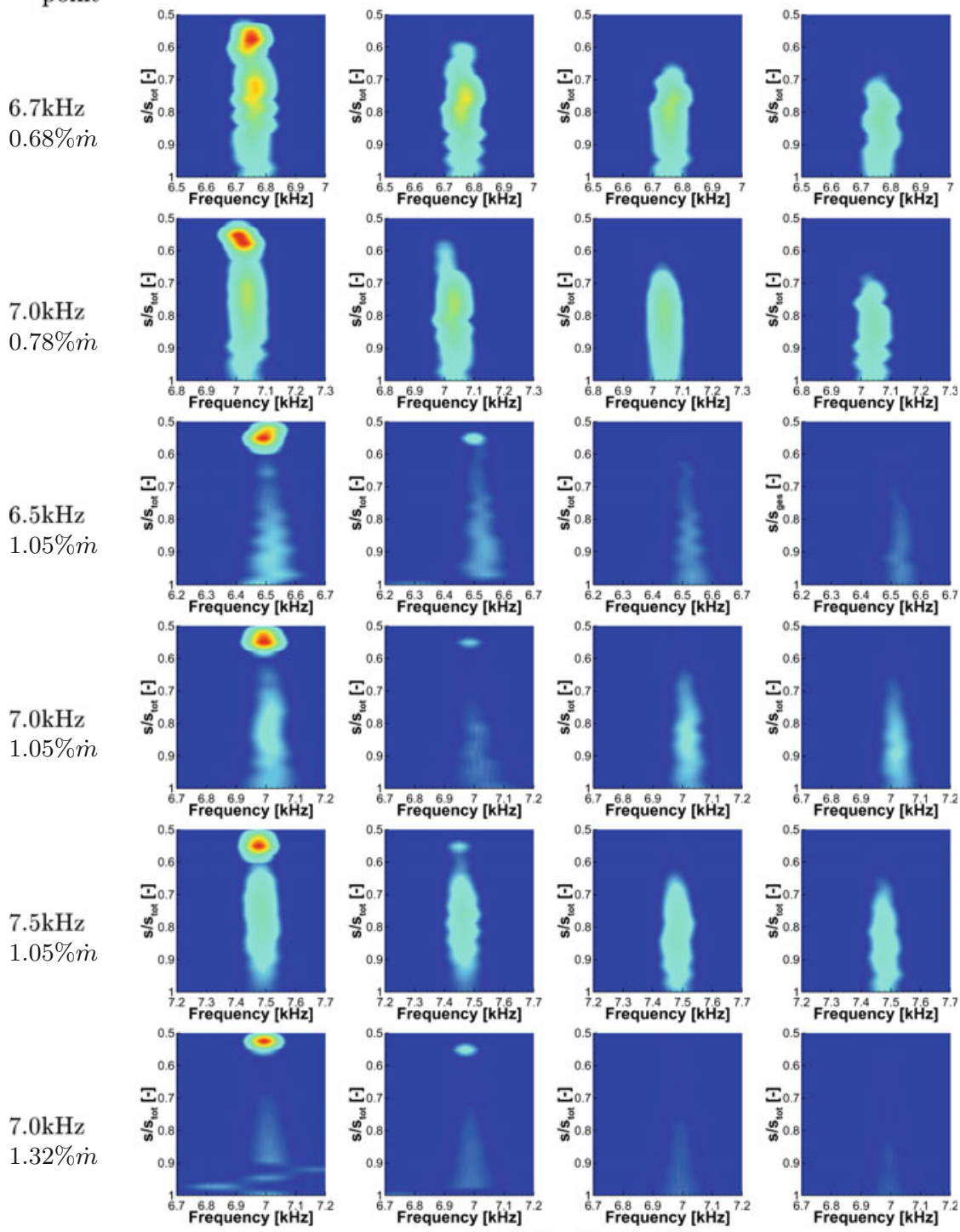

normalized PSD from Welch's method

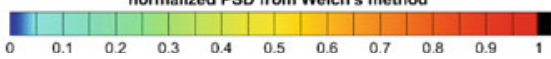

Fig. 10 Normalized PSD plots of the oscillator frequency from 1D hot-wire probe measurements: streamwise development at different distances to the surface 


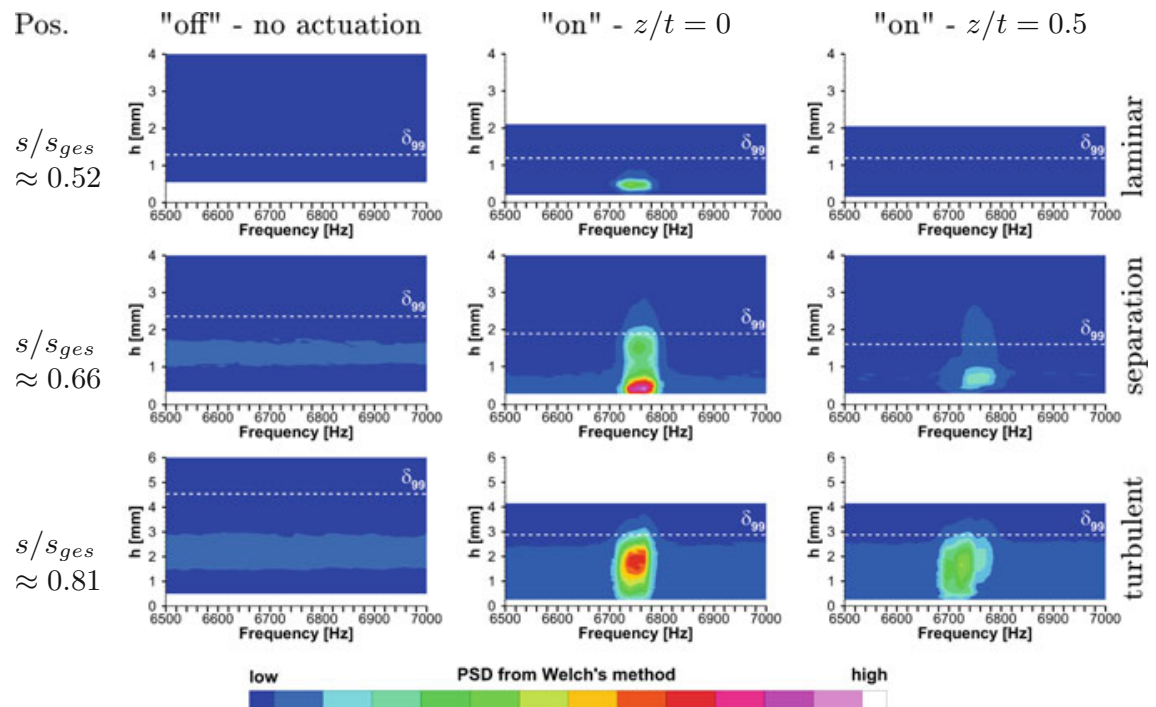

Fig. 11 PSD plots in the receptive frequency range of the investigated boundary layer flow with and without actuation: invested case $6.7 \mathrm{kHz}$ and $\dot{m}_{o s c} \approx 0.68 \% \cdot \dot{m}_{\text {pas }}$

intensity plots for actuator modes "off" as well as "on" for two different spanwise positions $z / t$. Positions straight downstream of one oscillator outlet are indicated with $z / t=0$ (center of the outlet), whereas measurements in the middle plane between two oscillator outlets are referred to as $z / t=0.5$. The spanwise spacing $t$ between two oscillator outlets is equal to $t=5.33 \mathrm{~mm}$, whereas the diameter of one outlet is $1 \mathrm{~mm}$. Three distinct positions in streamwise direction were chosen according to the boundary layer state with activated oscillator (laminar, separation, turbulentcompare Fig. 8). Each PSD plot shows the frequency range of $6.5-7 \mathrm{kHz}$ analogue to Fig. 10 in wall-normal direction $h$. The boundary layer thickness $\delta_{99}$ is indicated with the white dashed line in each plot. The wall-normal distance $h$ is re-scaled for the furthest downstream positions, equally adjusted for all three cases, to cover the full boundary layer thickness for the "off" case.

For the non-actuated case, increased PSD levels are only visible for the separated boundary layer within the shear layer (positions $s / s_{g e s} \approx 0.66$ and 0.81 ). The increased levels are present for the whole frequency range and therefore not related to any specific bandwidth. Taking a closer look at the PSD plots for the "on" case just downstream the oscillator outlet, the actuation frequency is visible only at $z / t=0$. Indications of the actuation frequency at the midspan positions $(z / t=0.5)$ can for the first time clearly correlated to the actuation at $s / s_{\text {ges }} \approx 0.66$. Furthermore, it is evident that due to the interaction within the boundary layer, the actuation frequency spreads throughout the whole boundary layer height. At the same time it is also restricted to it. At $s / s_{\text {ges }} \approx 0.81$ the character and extension in wall-normal direction are similar, the intensity, however, is slightly lower at the $z / t=0.5$ position. 
The interaction phenomena and the mixing process are very complex phenomena and are discussed in more detail in another paper by Bettrich et al. [15].

The development of the boundary layer thickness is strongly dependent on the state of the boundary layer. For the non-actuated case the boundary layer thickness increases rapidly when flow separation occurs, resulting in high total pressure losses. Since the momentum thickness increases as well, a thicker boundary layer can be correlated to an increase in loss behavior, too. This is the case for the boundary layer without actuation. However, with AFC activated, the boundary layer thickness remains thin with a moderate increase up to $s / s_{\text {ges }} \approx 0.66$. Further downstream, when transition over the small separation bubble occurs, the boundary layer thickness slightly increases. To preserve a thin boundary layer, AFC in the right frequency bandwidth and small induced momentum but strong interaction of the high frequency pulsed blowing with the boundary layer turns out to be favorable in terms of loss reduction. A target oriented AFC design cannot only control flow separation but also allows to control the boundary layer development, including the location of transition. Allowing a small separation bubble with defined and delayed turbulent reattachment turns out to be beneficial to reduce profile losses. The later transition occurs, the lower are the overall all losses, as long as the flow reattaches. Based on these results it is expected that there is further potential for loss reduction by reducing the mass flow. Decreased mass flow could delay the transition even further and will therefore potentially reduce turbulent boundary layer losses if interaction with the boundary layer still occurs. A mass flow reduction can be achieved either with scaled oscillators in size with the same frequency and/or an increased spacing of the oscillator outlet holes.

\section{Conclusions and Outlook}

The investigations on high frequency boundary layer actuation presented in this paper are carrying forward several promising investigations on flow control with the T161 cascade at the Institute of Jet Propulsion (ISA). With the use of fluidic oscillators, the aerodynamically highly loaded T161 low pressure turbine research profile showed a very significant reduction in the overall profile losses by $40 \%$. An even greater mass flow reduction (as low as $\dot{m}_{A F C}=2.1 \cdot 10^{-4} \cdot \dot{m}_{\text {pas }}$ ) was achieved for a turbine exit casing, which was specifically designed for utilization of active flow control (AFC). Motivated by these promising results, much potential for further improvements was expected. However, for investigations with the focus on the fundamental understanding of the interaction phenomena between high frequency pulsed blowing and the boundary layer, a new approach became necessary. For this purpose, a new experimental testbed was developed. In order to continue the previous work on the T161, its suction side flow was successfully reproduced on a flat plate with the same pressure distribution, induced by opposed contoured walls. All investigations were carried out at the High Speed Cascade Wind Tunnel at ISA. The results presented here are for the T161 equivalent operating point with Reynolds number of $R e=70,000$ (based 
on chord lenght), theoretical outflow Mach number of $M a_{2, t h}=0.6$ and inflow free stream turbulence level of $T u \approx 4 \%$.

The actuator used here for the flow control application is a specifically adapted and scaled design of the coupled fluidic oscillator, developed at ISA. It enables independent mass flow and frequency variations in a certain range. Based on preliminary studies, the receptive range of the boundary layer was estimated in order to ensure an effective actuator design. The frequency of the oscillator was varied between 6.5 and $7.5 \mathrm{kHz}$ and the mass flow rate between $\dot{m}_{\text {osc }} \approx 0.68$ and $1.32 \% \cdot \dot{m}_{\text {pas }}$. The different oscillator operating points are evaluated and compared according to their respective integral total pressure loss coefficients, transition behavior along the surface, and interaction with the boundary layer flow. Among these operating points the one with the lowest mass flow rates $\left(6.7 \mathrm{kHz}\right.$ and $\left.\dot{m}_{\text {osc }} \approx 0.68 \cdot \dot{m}_{\text {pas }}\right)$ turned out to be most efficient in terms of loss reduction and air consumption. Consequently it was chosen for more detailed investigations applying Preston probe, particle image velocimetry, and 1D hot-wire measurements.

\section{The main findings can be summarized as follows:}

1. A further upstream position of the oscillator outlets compared to the previous work on the T161 cascade is very beneficial in terms of effectiveness of the flow control concept. The invested mass flow could be reduced by $60 \%$.

2. Reducing the invested mass flow turns out to be beneficial, as long as the flow separation is under control. The reason for the lower integral losses can be explained by a delayed but controlled transition, which leads to a shorter streamwise extent of the turbulent boundary layer.

3. A change in actuation frequency within the estimated receptive range shows only minor impact on the boundary layer development and the associated loss generation. The control effect within the investigated range is found to be primarily dependent on the mass flow rate. However, actuation frequencies with weak boundary layer interaction show higher integral total pressure losses compared to cases with higher interaction and same mass flow rates.

4. Actuation within the estimated receptive range of the boundary layer indicates an increased interaction of the AFC with the boundary layer for decreased mass flow rates. High AFC mass flow rates turned out to be disadvantageous for the high frequency actuation to interact with the boundary layer. Higher mass flow rates are less effective in terms of transition control and loss reduction.

5. The key for further substantial increase in AFC effectiveness is to decrease the boundary layer thickness. Based on the results of this work this can be achieved most efficiently with an actuation in the receptive range to take advantage of the interaction between actuation and boundary layer flow. At the same time the momentum should be reduced to the extent that a controlled transition occurs as far downstream as possible. A Promising strategy would be down scaling the oscillators or increase their spacing.

With the current investigations on AFC with fluidic oscillators, substantial progress was achieved. The valuable results in the presented degree of details became 
possible with the newly developed experimental testbed, which will be used in upcoming investigations on high frequency boundary layer actuation even more extensively. The oscillator design in the estimated receptive frequency range shows for some cases a strong interaction with the boundary layer flow with promising loss characteristics. When all the governing effects are investigated even further, the authors are convinced that there is great potential to further reduce the mass flow investment, while keeping the control aspect effective, resulting in a very efficient fluidic oscillator design for AFC.

Acknowledgements The authors gratefully acknowledge the financial support of the German Research Foundation (Deutsche Forschungsgemeinschaft, DFG), which funded this research project (NI 586/9-1) on fundamental investigations of fluidic oscillators. The authors are also very grateful to the reviewers. Their valuable and critical comments contributed significantly to the quality of the final paper.

\section{References}

1. King, R., Heinz, N., Bauer, M., Grund, T., Nitsche, W.: Flight and wind-tunnel tests of closedloop active flow control. AIAA. J. Aircr. 50(5), 1605-1614 (2013). 10 pages. https://doi.org/ 10.2514/1.C032129

2. Niehuis, R., Mack, M.: Active boundary layer control with fluidic oscillators on highly-loaded turbine airfoils. In: King, R. (ed.) Active Flow and Combustion Control. Springer Series Notes on Numerical Fluid Mechanics and Multidisciplinary Design, vol. 127 (2014). (Invited Paper). https://doi.org/10.1007/978-3-319-11967-0_1

3. Cattafesta, L.N., Sheplak, M.: Actuators for active flow control. Ann. Rev. Fluid Mech. 43(1), 247-272 (2011). https://doi.org/10.1146/annurev-fluid-122109-160634

4. Dovgal, A.V., Kozlov, V.V., Michalke, A.: Laminar boundary layer separation: instability and associated phenomena. Progr. Aerosp. Sci. 30(1), 61-94 (1994). https://doi.org/10.1016/03760421(94)90003-5

5. Bons, J.P., Hansen, L.C., Clark, J.P., Koch, P.J., Sondergaard, R.: Designing low-pressure turbine blades with integrated flow control. In: ASME Turbo Expo GT2005-68962, Reno, pp. 1079-1091 (2005). 13 pages. https://doi.org/10.1115/GT2005-68962

6. Bons, J.P., Reimann, D., Bloxham, M.: Separated flow transition on an LP turbine blade with pulsed flow control. ASME J. Turbomach. 130(2), 021014 (2008), 8 pages. https://doi.org/10. $1115 / 1.2751149$

7. Bons, J.P., Pluim, J., Gompertz, K., Bloxham, M. und Clark, J.P.: The application of flow control to an AFT-loaded low pressure turbine cascade with unsteady wakes. ASME J. Turbomach. 134(3), 031009 (2011). 11 pages. https://doi.org/10.1115/1.4000488

8. Rist, U.: Instability and transition mechanisms in laminar separation bubbles. In: VKI/RTOLS, Low Reynolds Number Aerodynamics on Aircraft Including Applications in Emerging UAV Technology, Rhode-Saint-Genese (2003). http://citeseerx.ist.psu.edu/viewdoc/ download?doi=10.1.1.638.6411\&rep=rep1\&type=pdf

9. Mack, M., Brachmanski, R., Niehuis, R.: The effect of pulsed blowing on the boundary layer of a highly loaded low pressure turbine blade. ASME Turbo Expo GT2013-94566, San Antonio, p. V06AT36A015 (2013). 10 pages. https://doi.org/10.1115/GT2013-94566

10. Kurz, J., Hoeger, M. und Niehuis, R.: Design of a highly loaded turbine exit case airfoil with active flow control. In: ASME IMECE2016-66008, ASME IMECE, Phoenix, p. V001T03A049 (2016). 11 pages. https://doi.org/10.1115/IMECE2016-66008 
11. Kurz, J., Hoeger, M., Niehuis, R.: Active boundary layer control on a highly loaded turbine exit case profile. In: ETC12, ETC2017-191, Stockholm (2017). http://www.euroturbo. eu/publications/proceedings-papers/etc2017-191/

12. Kurz, J., Hoeger, M., Niehuis, R.: Influence of active flow control on different kinds of separation bubbles. In: Proceedings of the XXIII. ISABE, Manchester, ISABE-2017-22572 (2017)

13. Mack, M., Niehuis, R., Fiala, A.: Parametric study of fluidic oscillators for use in active boundary layer control. In: ASME Turbo Expo GT2011-45073, Vancouver, pp. 469-479 (2011). 11 pages. https://doi.org/10.1115/GT2011-45073

14. Mack, M., Niehuis, R., Fiala, A., Guendogdu, Y.: Boundary layer control on a low pressure turbine blade by means of pulsed blowing. ASME J. Turbomach. 135(5), 051023 (2013). 8 pages. https://doi.org/10.1115/1.4023104

15. Bettrich, V., Bitter, M., Niehuis, R.: Interaction phenomena of high frequency pulsed blowing in LP turbine-like boundary layers at high speed test conditions. In: ASME Turbo Expo 2018 GT2018-75475, Oslo (2018)

16. Bettrich, V., Niehuis, R.: Experimental investigations of a high frequency master-slave fluidic oscillator to achieve independent frequency and mass flow characteristics. In: ASME IMECE 2016-66782, Phoenix, p. V001T03A061 (2016). https://doi.org/10.1115/IMECE2016-66782

17. Schlichting, H., Gersten, K.: Boundary-Layer Theory. Springer, Heidelberg (2017). https://doi. org/10.1007/978-3-662-52919-5

18. Simoni, D., Ubaldi, M., Zunino, P., Lengani, D., Bertini, F.: An experimental investigation of the separated-flow transition under high-lift turbine blade pressure gradients. Flow Turbul. Combust. 88(1-2), 45-62 (2012). https://doi.org/10.1007/978-3-319-11967-0_1

19. Sturm, W., Fottner, L.: The high-speed cascade wind tunnel of the German armed forces university Munich. In: 8th Symposium on Measuring Techniques for Transonic and Supersonic Flows in Cascades and Turbomachines, Genova, Italy (1985)

20. Wazzan, A.R., Okamura, T.T., Smith, A.M.O.: Spatial and temporal stability charts for the Falkner-Skan boundary-layer profiles. REPORT NO. DAC-67086, McDonnell Douglas Astronautics Company-Huntington Beach (1968)

21. Walker, G.J., Gostelow, J.P.: Effects of adverse pressure gradients on the nature and length of boundary layer transition. ASME J. Turbomach. 112(2), 196-205 (1990). 10 pages. https:// doi.org/10.1115/1.2927633

22. Stotz, S., Wakelam, C.T., Niehuis, R., Guendogdu, Y.: Investigation of the suction side boundary layer development on low pressure turbine airfoils with and without separation using a Preston probe. In: ASME Turbo Expo GT2014-25908, Duesseldorf, p. V02CT38A025 (2014). 13 pages. https://doi.org/10.1115/GT2014-25908

23. Kaehler, C.J., Scholz, U., Ortmanns, J.: Wall-shear-stress and near-wall turbulence measurements up to single pixel resolution by means of long-distance micro-PIV. Exp. Fluids 41(2), 327-341 (2006). https://doi.org/10.1007/s00348-006-0167-0 